\title{
Impedance alterations in healthy and diseased mice during electrically-induced muscle contraction
}

\author{
Benjamin Sanchez, Jia Li, Tom Geisbush, Ramon Bragos Member, IEEE and Seward B Rutkove Member, IEEE
}

\begin{abstract}
Alterations in the health of muscles can be evaluated through the use of electrical impedance myography (EIM). To date, however, nearly all work in this field has relied upon the measurement of muscle at rest. To provide insight into the contractile mechanisms of healthy and disease muscle, we evaluated the alterations in the spectroscopic impedance behavior of muscle during the active process of muscle contraction. The gastrocnemii from a total of 13 mice were studied ( 5 wild type, 4 muscular dystrophy animals, and 4 amyotrophic lateral sclerosis animals). Muscle contraction was induced via monophasic current pulse stimulation of the sciatic nerve. Simultaneously, multisine EIM (1 $\mathrm{kHz}$ to $1 \mathrm{MHz}$ ) and force measurements of the muscle were performed. Stimulation was applied at 3 different rates to produce mild, moderate and strong contractions. We identified changes in both single and multifrequency data, as assessed by the Cole impedance model parameters. The processes of contraction and relaxation were clearly identified in the impedance spectra and quantified via derivative plots. Reductions in the center frequency $f_{c}$ were observed during the contraction consistent with increasing muscle fiber diameter. Different EIM stimulation rate-dependencies were also detected across the 3 groups of animals.
\end{abstract}

Index Terms-dynamic electrical impedance myography, muscle, isometric contraction, time-varying impedance, multisine excitations.

\section{INTRODUCTION}

$\mathbf{W}$ EAKNESS remains the major source of disability of most diseases that affect the peripheral nervous system and muscle. Many of these disorders, which include conditions such as amyotrophic lateral sclerosis, muscular dystrophy, inflammatory myopathies, and certain polyneuropathies, are without effective therapy [1]. Fortunately, thanks to an improved understanding into the pathogenesis of these diseases and creative new therapeutic strategies, discoveries are beginning to yield concrete advances and certain diseases, previously thought to be untreatable, such as Duchenne muscular dystrophy, are now finding potential effective therapies

B. Sanchez, J. Li, T. Geisbush and S. B. Rutkove are with the Department of Neurology, Division of Neuromuscular Diseases, Beth Israel Deaconess Medical Center, 330 Brookline Avenue, Harvard Medical School, Boston, MA 02215-5491, USA. Phone: 617-667-8130. Email: bsanchez@bidmc. harvard.edu

R. Bragos is with the Electronic Engineering Department, Ed.C4 Campus Nord, Universitat Politecnica de Catalunya (UPC), Jordi Girona 1-3, 08034, Barcelona, Spain.

This research was supported in part by Redes de Investigacion del Instituto Carlos III (REDINSCOR, RD06/0003) and Fondo Europeo de Desarrollo Regional (FEDER), and in part by grant R01 NS055099 to the Beth Israel Deaconess Medical Center General Clinical Research Center from the National Institute of Health.

Copyright (c) 2013 IEEE. Personal use of this material is permitted. However, permission to use this material for any other purposes must be obtained from the IEEE by sending an email to pubs-permissions@ieee.org.
[2]. Still, a great deal of work needs to be accomplished if the weakness produced by these diseases is to be effectively ameliorated.

How each of these conditions actually produces muscle weakness, however, is often not fully understood. Indeed, we are very capable of measuring the contractile process up to and including the point of the electrical depolarization of the muscle [3]. We are also very capable of measuring the force output of the muscle itself. However, we are bereft of any technique for studying the actual mechanical process of contraction in vivo, even though the basic physiology of the excitation-contraction coupling process is well understood. For example, with standard electrophysiological techniques, such as nerve conduction studies and electromyography, we can measure action potential propagation down the nerve, across the neuromuscular junction, and the electrical depolarization of the muscle fibers themselves [3]. With needle electromyography we can evaluate membrane stability, the health of individual motor units and changes in the electrical properties of the muscle [4]. Imaging modalities may also be potentially useful for assessing muscle contraction. For example, ultrasound has been studied preliminarily in this regard [5]. However, the time constants for data acquisition using magnetic resonance imaging, which may have the potential of showing exquisite structural detail with contraction, are too long to make such an application feasible. One technique that could provide new insights into the actual mechanics of muscle contraction is that of electrical impedance myography (EIM). EIM is a tetrapolar bioimpedance-based technique in which localized single-frequency/spectroscopy measurements of muscle are made and the impedance measures are used to assess muscle health [6]. To date, EIM measures in static muscle (contracted or relaxed) have been shown to be sensitive to the presence and severity of neuromuscular disorders [7][9]. Indeed, EIM has been proposed as a new tool to monitor disease status and effects of therapy in amyotrophic lateral sclerosis [10]. However, only a single pilot study has attempted to evaluate the alterations of impedance change with muscle contraction [11], what we have called dynamic EIM. In that work, human subjects were asked to exert voluntary isometric gripping force against a hand dynamometer while sensingelectrodes on the forearm recorded impedance shifts at 50 $\mathrm{kHz}$. While representing a good starting point, this work was limited by (1) measurements at a single frequency and (2) limited time-synchronization as the contraction was performed voluntarily rather than via direct neuronal stimulation.

In this paper, we show the feasibility of performing timevarying impedance measurements of skeletal muscle in healthy 
and diseased mice during electrically-induced muscle contraction. To perform simultaneous EIM spectroscopy measurements $(1 \mathrm{kHz}$ to $1 \mathrm{MHz})$, multisine impedance spectroscopy is used [12]. To fully achieve time-synchronization, both the force exerted by the muscle and the nerve stimuli applied are recorded simultaneously to the muscle impedance measurement. We identify novel alterations in the impedance characteristics of muscle with contraction with potentially important implications for future animal and human research.

\section{MATERIALS AND METHODS}

\section{A. Animals}

All studies were approved by the Beth Israel Deaconess Medical Center Institutional Animal Care and Use Committee. All animals obtained from Jackson Laboratories (Bar Harbor, Maine) were allowed to acclimate for 48 hours prior to use and fed ad libitum. 5 wild type (WT) mice, 4 amyotrophic lateral sclerosis (ALS) mice and 4 muscular dystrophy (mdx) mice were studied.

\section{B. Animal set up}

Animals underwent all measurements under 1-2\% inhaled isoflurane anesthesia delivered by nosecone, with body and muscle temperature being maintained by a heating pad. The basic technique was similar to that previously described, except that gastrocnemius rather than extensor digiotirum longus was studied [13]. Briefly, the skin overlying both left and right hind-limbs was cut with a scissors and the tissue dissected down to expose the biceps femoris muscle, which was removed proximally. The calcaneal tendon was cut at its insertion point, and dissected away from the underlying fascia and soleus muscle. The cut end was then tied to a piece of silk suture material which was then connected to the force measuring system. Animal sacrifice was performed via the use of FatalPlus ${ }^{\circledR}$ at the completion of measurements.

\section{Force measurement}

The length of muscle contraction was measured using an external spring attached to a load cell (model FT 10E 50199-4) that was run through a P11T amplifier and RPS210 power supply (Grass Technologies West Warwick, RI). PT11 frequency response was set at $30 \mathrm{~Hz}$ and has an output impedance of 500 $\Omega$. The load cell was calibrated to produce an analog signal of $39.75 \mathrm{~V} \mathrm{~N}^{-1}$. The external spring was placed in series with the beam shaft of the force transducer and the muscle preparation (see Figure 1). External spring length was kept constant at $3.5 \mathrm{~cm}$ between the beam shaft and the relaxed muscle. This external spring length was relatively large compared to the length of muscle contraction during stimulation, allowing for the muscle to essentially work with a constant force throughout contraction. Upon stimulation, the gastrocnemius was allowed to freely contract and the force exerted on the spring was recorded.
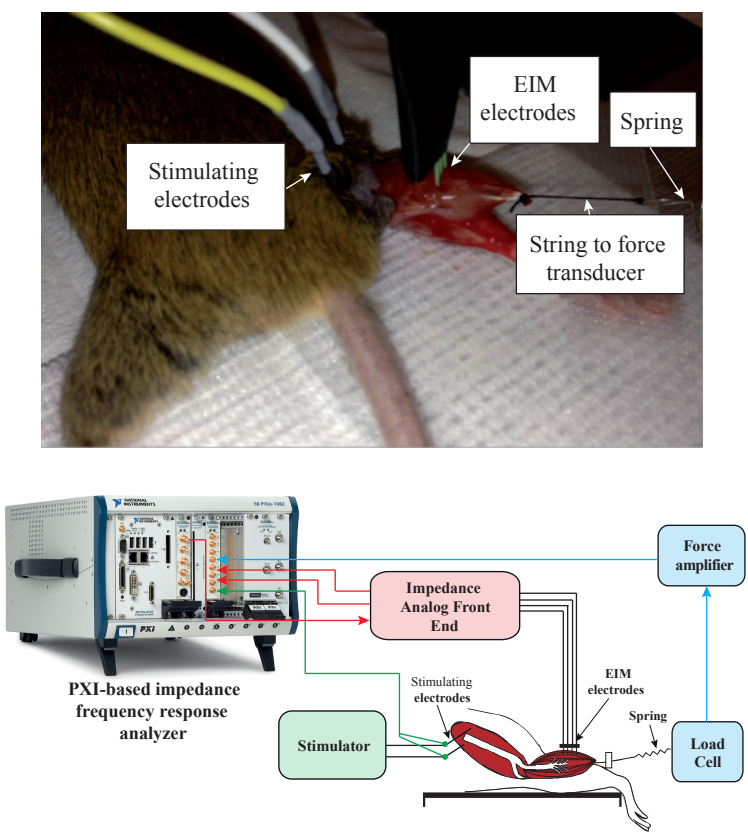

Fig. 1. (top) Picture of the animal experimentation; (bottom) Sketch of the experimental setup. The PXI-based frequency response analyzer acquires synchronously the force exerted by the gastrocnemius, the EIM and the stimuli applied to the sciatic nerve.

\section{Nerve stimulation}

To ensure that the same amount current was applied to each hind leg of each animal during nerve stimulation, the compound motor action potential (CMAP) was identified first using a TECA Synergy T2 EMG Monitoring System (Viasys, Madison, WI). The CMAP was recorded via disposable ring electrodes (Product 019-435500, Faith Medical Inc., Steedman, MO) around the entire leg with stimulating the sciatic nerve at the sciatic notch (Neuroline 746 12-100/25 needle electrodes, Ambu, Denmark) [14]. The specific current which was used to obtain the CMAP was then used for the following stimulation paradigms: 3 pulses at $9 \mathrm{~Hz}$ (weak contraction) 6 pulses at $18 \mathrm{~Hz}$ (moderate contraction), and 10 pulses at 30 $\mathrm{Hz}$ (strong contraction). By choosing these frequencies and number of stimuli, the overall duration of the contract remained constant at 0.33 seconds. In all stimulation paradigms, the amplitude and duration of the monophasic pulses was set to $8-10 \mathrm{~mA}$ and $0.2 \mathrm{~ms}$ respectively.

\section{E. Impedance measurement}

The muscle impedance was measured using a custom broadband impedance frequency response analyzer (FRA) [12], [15], [16]. The impedance measurement system was implemented on a rugged PC-platform PXI (PCI eXtensions for Instrumentation) platform from National Instruments. The FRA included an embedded controller PXIe-8130, a 2 channel high-speed digitizer card PXIe-5122 (100 Ms s-1, 64 MB/channel, 14 bits), a 8-channel digitizer card PXI-5105 (60 $\mathrm{Ms} \mathrm{s}^{-1}, 128$ $\mathrm{MB}, 12$ bits) and an Arbitrary Waveform Generator (AWG) card PXI-5422 (200 Ms s ${ }^{-1}, 32 \mathrm{MB}, 16$ bits). Synchronously to the reference excitation generated by the PXI-5422, the 
PXIe-5122 acquired both the noisy current- voltage observations coming from the analog front end used to interface the FRA with the electrodes. The FRA analog front end consisted of a drive buffer (OPA656) and differential amplifier (AD830) for the high and the low potential terminals and a currentto-voltage converter (OPA656) for the low current sensing terminal [17].

Four disposable monopolar EMG needle electrodes of Viasys Healthcare (Ref 902-DMG50) were used as needle electrodes. The four strips were attached to a metal plate (30 $\mathrm{mm} \mathrm{L} \mathrm{x} 15 \mathrm{~mm} \mathrm{~W}$ ) in a line with an inter-electrode separation distance of $1 \mathrm{~mm}$ (center to center).

For each leg, the tetrapolar electrode array was placed transverse $(90 \mathrm{deg})$ to the major muscle fiber direction determined by visual inspection. Data is shown in Section III.

\section{F. Data acquisition and analysis}

The reference excitation generated with the PXI-5422 to measure impedance was a random phase multisine signal, with $T_{\mathrm{ms}}=1 \mathrm{~ms}$ periodicity. The amplitude the harmonics were chosen to be equal (flat multisine), the phases were randomly chosen in $[0,2 \pi), k \in \mathbb{K}_{\mathrm{exc}}$ was the set of excited frequencies (26 quasi-logarithmic frequencies distributed from $1 \mathrm{kHz} \rightarrow$ $939 \mathrm{kHz}) ; f_{s}=5 \mathrm{MHz}$ being the sampling frequency. To avoid spectral leakage, an integer number of periods of the multisine excitation was measured and the entire measurement setup was synchronized with the reference multisine signal. The current as well as the voltage impedance responses were passed through anti-Alias filters (1 $\mathrm{MHz}$ cutoff frequency) before the signals were sampled with the PXIe-5122, such that aliasing was also avoided.

Data streaming from the PXIe-5122 to the PXIe-8130 controller hard-disk was performed to store in real time the sampled data. To stream-data-to-disk, the two main bus- and processor-intensive tasks were: 1) acquiring data from the PXIe-5122 and 2) writing data to a binary file directly to the hard disk. To better utilize processor resources, LabVIEW processes were divided into two independent loops (multithreading) using a queue structure (producer-consumer structure).

Once the sampled noisy voltage and current observations were available to be processed, the impedance spectrum was computed using Matlab [18]. The signal linear time-invariant signal processing tool used to estimate the impedance was based on the short time Fourier transform (STFT) using overlapping records. The spectral leakage was intentionally avoided by setting the STFT window duration (i) by processing an integer number of periods of the reference for each record and (ii) by using a rectangular time sliding window [19], [20]. For the periodic overlapped segment averaging, as this is the case, the (noncalibrated) voltage-current spectra were calculated as

$$
X(k)=\frac{1}{\sqrt{N}} \sum_{n=0}^{N-1} x[n] e^{-j 2 \pi k n \frac{1}{N}},
$$

with $X=\{V, I\}$ the Discrete Fourier Transform (DFT) spectra, $x=\{v, i\}$ the noisy voltage-current observations; $N$ the samples length of the record processed and $T_{s}=\frac{1}{f_{s}}$ the sampling interval.

A consistent impedance spectrum estimator $\widehat{Z}\left(\omega_{k}\right)$ (when $P \rightarrow \infty)$ is obtained from the division of the voltage-current sample mean Fourier coefficients at the excited frequencies [16], namely

$$
\widehat{Z}\left(\omega_{k}\right)=\frac{\widehat{V}(k)}{\widehat{I}(k)}=\frac{\frac{1}{P} \sum_{p=1}^{P} V^{[p]}(k)}{\frac{1}{P} \sum_{p=1}^{P} I^{[p]}(k)},
$$

with $\omega_{k}=2 \pi \frac{k}{T_{\mathrm{ms}}},\left\{V^{[p]}, I^{[p]}\right\}$ the excited DFT bins of the $p$-th period of the current-voltage signals. The impedance spectrum noise variance on $\widehat{Z}\left(\omega_{k}\right)$ was also estimated according to [21]

$$
\widehat{\sigma}_{\widehat{Z}}^{2}(k)=\left|\widehat{Z}\left(\omega_{k}\right)\right|^{2}\left(\frac{\widehat{\sigma}_{\widehat{V}}^{2}(k)}{|\widehat{V}(k)|^{2}}+\frac{\widehat{\sigma}_{\widehat{I}}^{2}(k)}{|\widehat{I}(k)|^{2}}-2 \operatorname{Re}\left(\frac{\widehat{\sigma}_{\widehat{V} \widehat{I}}^{2}(k)}{\widehat{\widehat{V}}(k) \widehat{\widehat{I}(k)}}\right)\right),
$$

with $\widehat{\sigma}_{\widehat{X_{1}} \widehat{X}_{2}}^{2}(k)$ the (co-)variance of the sample mean $\{\widehat{V}, \widehat{I}\}$ at the excited frequencies given by

$$
\widehat{\sigma}_{\widehat{X_{1}} \widehat{X_{2}}}^{2}(k)=\frac{1}{P(P-1)} \sum_{p=1}^{P}\left(X_{1}^{[p]}(k)-\widehat{X_{1}}(k)\right) \overline{\left(X_{2}^{[p]}(k)-\widehat{X_{2}}(k)\right)},
$$

with $X_{\{1,2\}} \in\{V, I\}$, where the shorthand notation $\sigma_{X_{i} X_{i}}^{2}=\sigma_{X}^{2}$ is adopted. For further details on the signal processing, the reader is referred to [16].

Ultimately, the impedance was calibrated by measuring a reference resistor (199 $\Omega, 1 \%)$. The calibration experiment was carried out at the same measurement frequencies using the same measurement setup, such that the effects of the cables and the amplifier response on the measurement error were compensated.

For processing the data with the STFT-based technique, we chose a $10 \mathrm{~ms}$ window duration $\left(T_{w}=10 \mathrm{~ms}\right)$, such that it contained $N_{\mathrm{ms}}=T_{\mathrm{ms}} T_{s}=5000$ samples per period. A total of $P_{\mathrm{ms}}=T_{w} T_{\mathrm{ms}}=10$ multisine periods fitted in each block/segment, such that the number of samples within $T_{w}$ was $N_{w}=P_{\mathrm{ms}} N_{\mathrm{ms}}=5 \times 10^{4}$ samples. The measurement lasted for $T=1$ seconds, giving a total of $N=T f_{s}=5 \times 10^{6}$ samples the length of the voltage and current signals. Furthermore, the selected fraction of overlap $(O)$ between segments was set to $60 \%$, such that $O=0.6 N_{w}=3 \times 10^{4}$ overlapping samples were used. In all, a total of 248 impedance spectra (26 frequencies) were processed in the $0.988 \mathrm{~s}$ recording period.

The force signal and the stimuli applied to the nerve were acquired with the PXI-5105 sampling at $1 \mathrm{Ms} \mathrm{s}^{-1}$ and then downsampled to $1 \mathrm{kHz}$ by averaging. High-frequency noise was filtered in Matlab with a low pass Butterworth filter.

\section{G. Data modeling}

The four Cole impedance model parameters were obtained by fitting the impedance data to the Cole impedance model [22] using a weighted complex nonlinear least square (WCNLS) method [23]. The estimated impedance spectrum noise from (2) was used as weight factors to obtain unbiased Cole parameters. The number of maximum iteration and function evaluations was set to $10^{6}$. The desired tolerance was set 

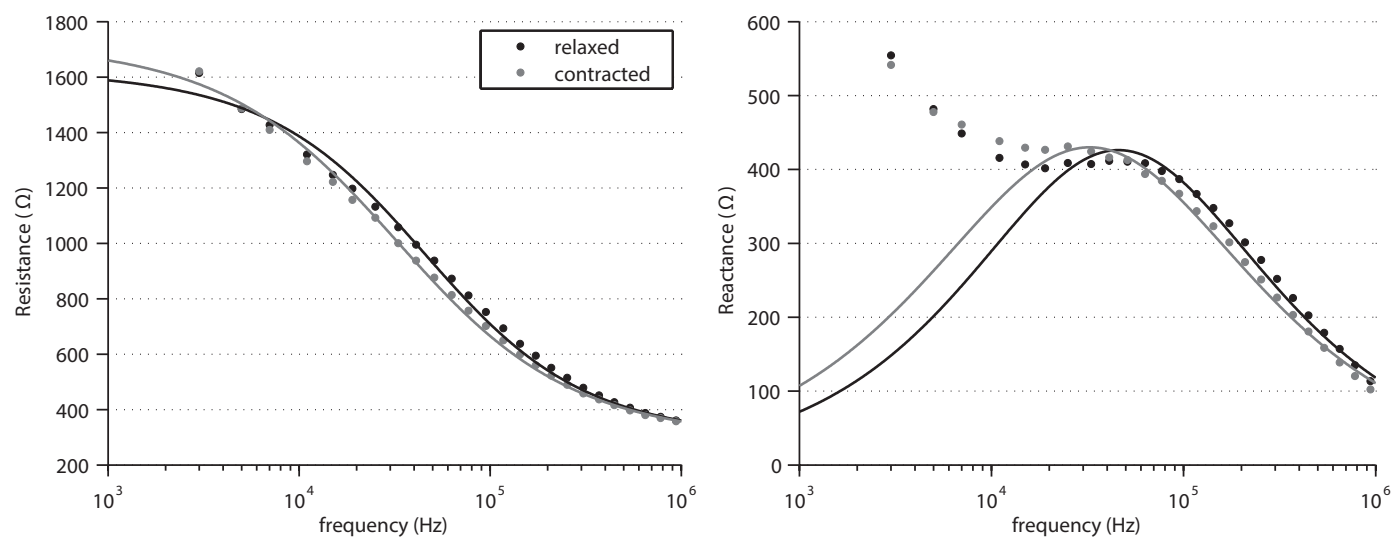

Fig. 2. Measured (circles) and Cole model (lines) resistance and reactance spectra for relaxed and contracted in normal animal. The electrodes were placed in transverse direction to the muscle fibers.

to $10^{-12}$. The initial values for $R_{0}$ and $R_{\infty}$ parameters were given from the initial and ending points of the impedance magnitude spectrum measured. The initial central frequency $f_{\mathrm{c}}$ was derived from the center frequency measured, and the $\alpha$ parameter was initially set to 0.5 . The algorithm used was Levenberg-Marquardt [24]. The numerical Jacobian matrix was finally computed to estimate the standard errors of the Cole impedance parameters.

\section{H. Muscle strength measurement}

The hind paw grip strength of each animal was measured separately prior these experiments via a Grip Strength Meter Single Computerized Sensor with Standard Pull Bars for Mice (CAT 1027CSM) (Columbus Instruments). While the animal was awake, the investigator (JL) held the animal at the base of the fore limbs and pulled away the animal from a small bar where its hind paws grasped. The maximum force out of 5 measurements was recorded.

\section{EXPERIMENTAL RESULTS}

\section{A. Muscle impedance spectroscopy data in normal animals during contraction}

Figure 2 shows the difference of the overall spectral changes during contraction for a WT animal. As can be seen, during contraction there is a shift in both resistance and reactance values, likely corresponding to the increasing size of membrane content between the measuring electrodes, more marked in frequencies below $100 \mathrm{kHz}$. Simply put, the increase in cellular and membrane content between the electrodes is the likely explanation for this observation. Also note the slight shift to the right where the maximum reactance occurs. In all the plots shown, the (capacitive) reactance of the impedance is multiplied by -1 for convention.

Of note, the low frequency data $(<10 \mathrm{kHz})$ in Figure 2 was not taken into account in the Cole data modeling as it was influenced by (1) the dynamics of the electrode impedance and (2) the nerve stimulating signal (refer to Section IV for further details). Indeed, the standard errors in the Cole model parameters shown in gray in Figure 4 and 7 below confirm that measurement errors were not propagated and the modeling was performed correctly [25].

Figure 3 shows the temporal evolution of the resistance, reactance, and force as well as the corresponding stimuli that were used to produce the muscle contraction, evaluated at a frequency of $51 \mathrm{kHz}$. The right side of Figure 3 shows derivative plots of that data. Of note, the force derivative data is noisy due to the very low voltage values recorded. At this stage, we did not filter the force data in order to maintain the integrity of the temporal response. One can see that force show similar changes during contraction as well as relaxation whereas the resistance and reactance plots reveal a temporal asymmetry of a normal contraction, with relaxation being a slower, less-well-defined event.

\section{B. Temporal evolution of the Cole impedance parameters during contraction in normal animals}

Figure 4 shows the temporal evolution of the Cole impedance parameters $\left\{R_{0}, R_{\infty}, f_{\mathrm{c}}, \alpha\right\}$ for a healthy WT mouse with two separate stimulation paradigms, each lasting approximately 0.33 seconds. On the left side, 6 stimuli are given in that 0.33 second time period; on the right side, 10 stimuli are given in that same time period, thus producing two contractions of varying strength (the right side contraction is approximately 4 times stronger than the left).

Overall, the changes in the $R_{0}$ and $R_{\infty}$ parameters both increasing are consistent with the expected changes, with an increase in extracellular volume over all tissue volume during contraction. Furthermore, it can be observed there is a noticeable decrease in the center frequency, most notable at the stronger stimulation rate, likely relating to the increase in the muscle fiber diameter during contraction. Moreover, the initial and final value of the parameters are different in both cases analyzed, suggesting that they can be used as an indicator of persistent alterations in the muscles properties in the time immediately following the completion of a muscle contraction. In addition, the reduction in $\alpha$, suggests reduced uniformity in muscle fiber size during contraction. Such alterations in normal animals suggest that such testing paradigms may reveal important and useful information in diseased states where the contractile process is impaired. 
This article has been accepted for publication in a future issue of this journal, but has not been fully edited. Content may change prior to final publication. Citation information: DOI 10.1109/TBME.2014.2320132, IEEE Transactions on Biomedical Engineering

IEEE TRANSACTIONS ON BIOMEDICAL ENGINEERING

(A) Impedance data over time
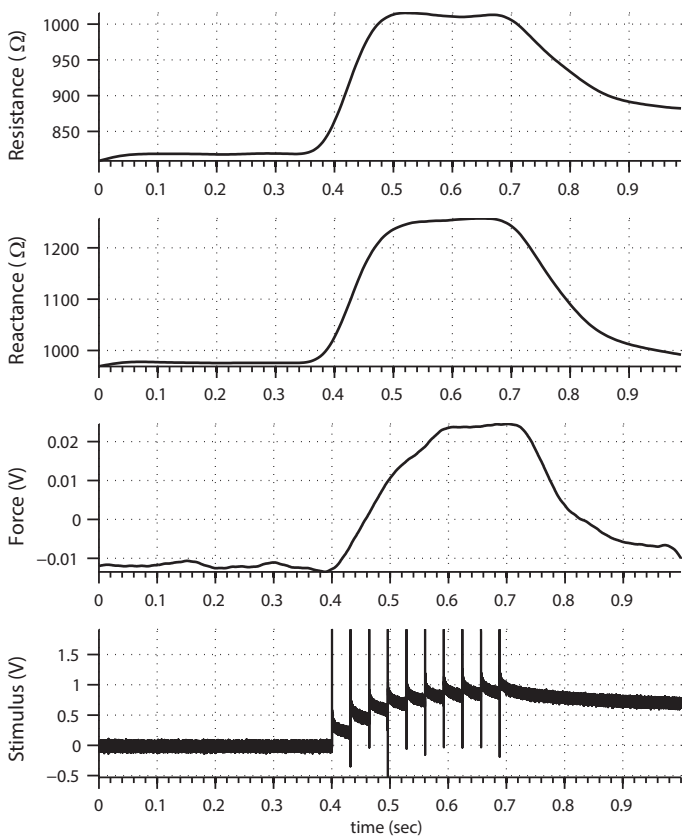

(B) Derivatives of the data
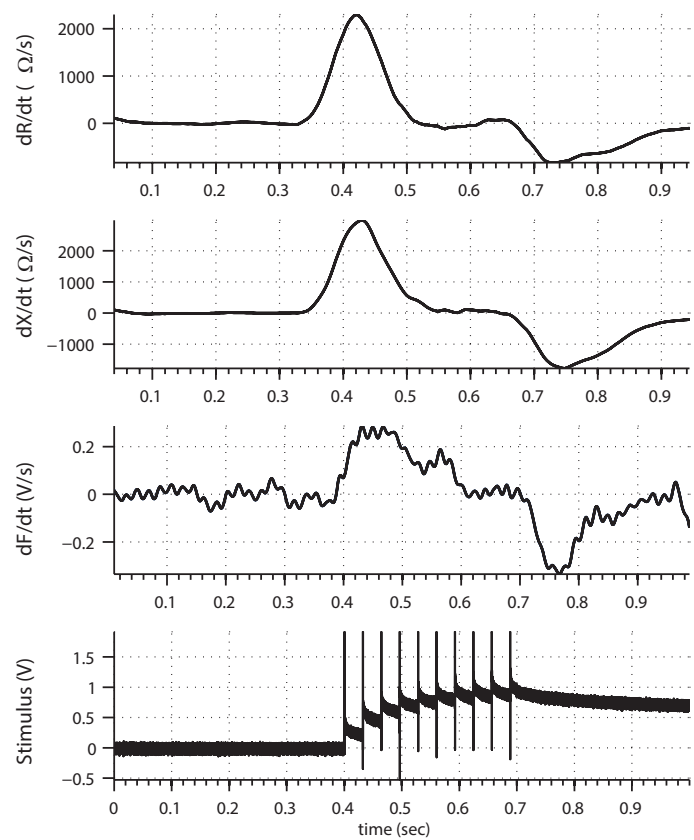

Fig. 3. (left) Time dependences of resistance (R), reactance (X) at the selected frequency of $51 \mathrm{kHz}$ and force (F) synchronized with the stimuli for normal mouse and their corresponding derivatives $\mathrm{dR} / \mathrm{dt}, \mathrm{dX} / \mathrm{dt}$ and $\mathrm{dF} / \mathrm{dt}$ (right).

(A) Rate of stimuli $18 / 6$
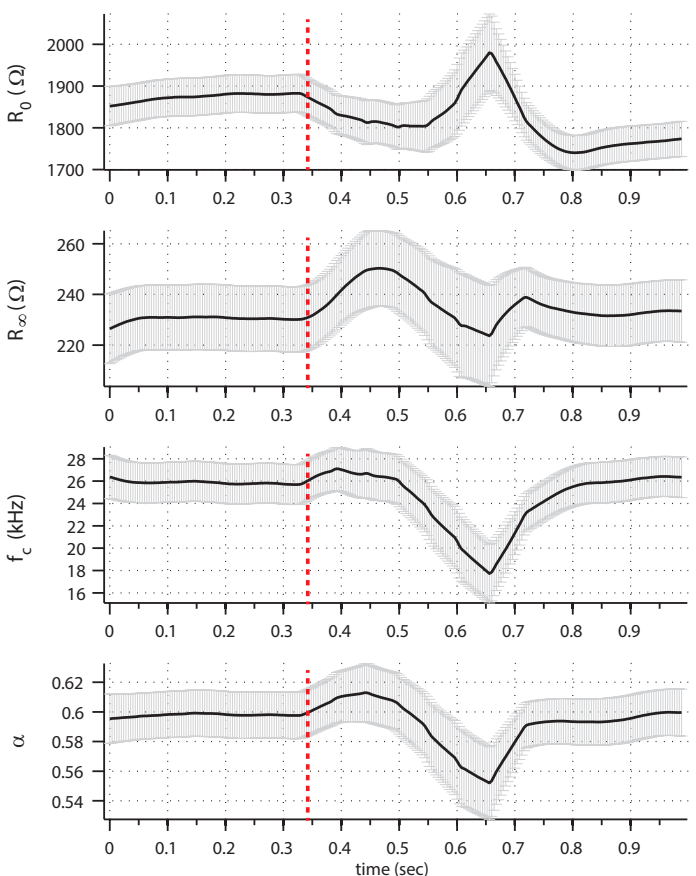

(B) Rate of stimuli $30 / 10$
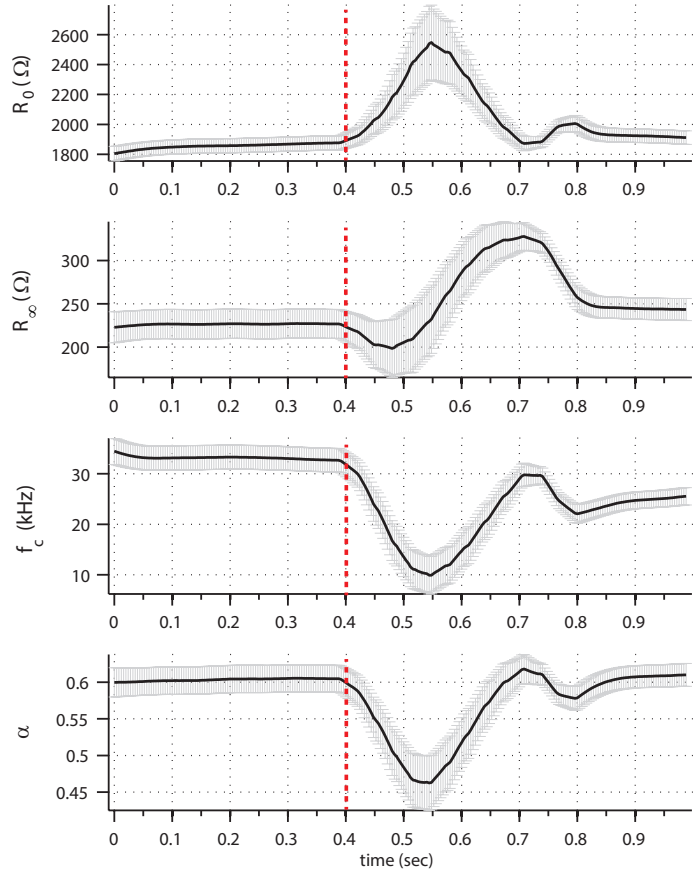

Fig. 4. Evolution of the Cole parameters during contraction-relaxation for two different experiments on the same normal animal. In gray, the Cole parameters standard errors corresponding to this particular animal (see Section II-G). The red dotted vertical line denote the onset of stimulation.

\section{Muscle impedance spectroscopy in ALS and mdx animals}

Figure 5-6 show data for an ALS animal and an mdx animal undergoing similar stimulation and recording paradigms as described for the WT animals in the previous section and are analogous to Figure 2 and Figure 3, shown for the WT animals. As can be seen in Figure 5, the resistance and reactance spectra show similar overall changes compared to the WT animals.
This is further confirmed by the similarity of the exerted force for each of the ALS and mdx animals analyzed w.r.t. the WT.

\section{Temporal evolution of the Cole impedance parameters during contraction in diseased animals}

Figure 7 shows the spectroscopic Cole parameters for the same mdx and ALS animals shown in Figure 5; this figure is 
(A) ALS
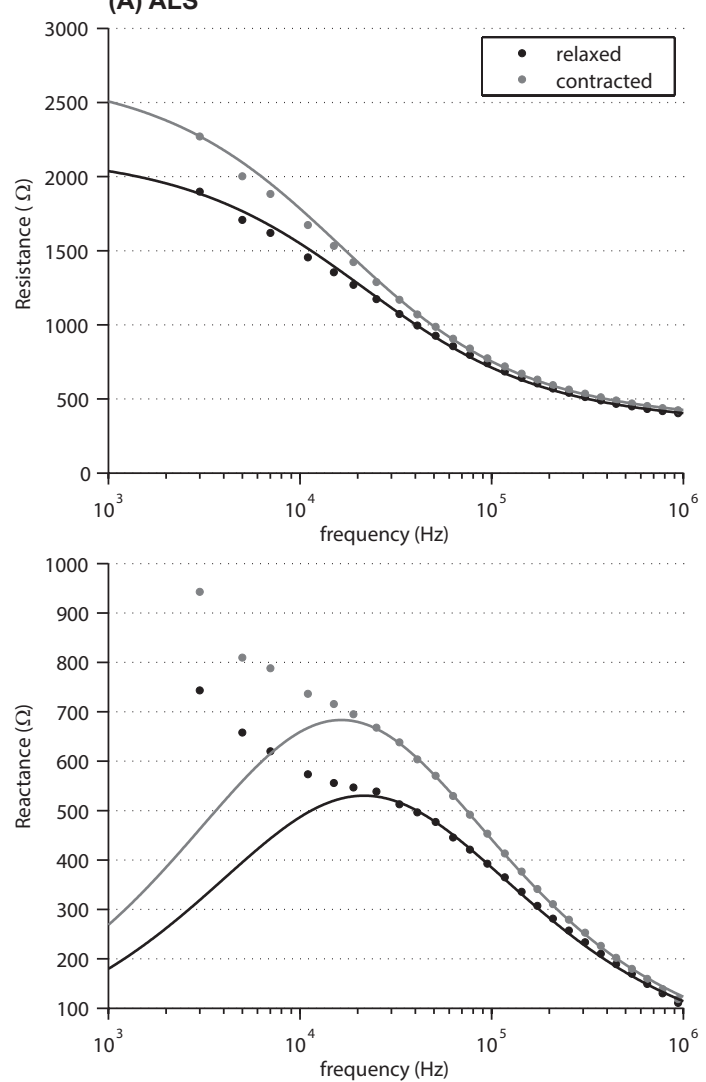

(B) $\mathrm{mdx}$
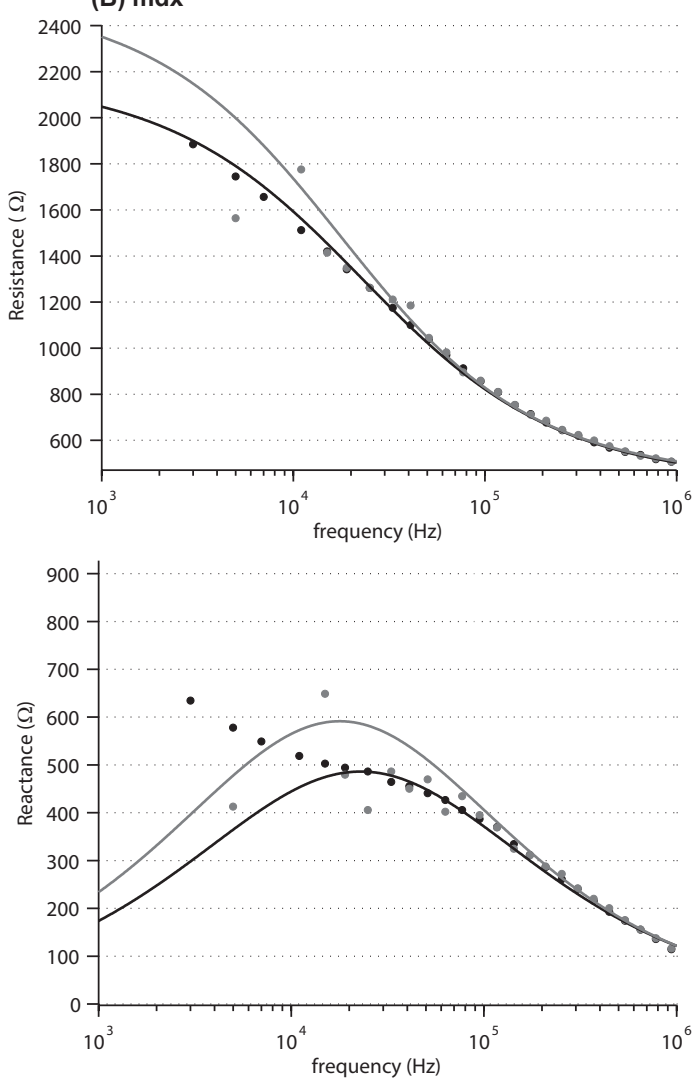

Fig. 5. Relaxed (black) and contracted (gray) resistance and reactance spectra for ALS (A) and mdx (B) animals; measured (circles) and Cole modeling data (lines). Data obtained from a $10 \mathrm{~ms}$ measurement.

analogous to Figure 4 shown for the WT animal. As can be seen, the alterations in the Cole parameters both between the mdx and ALS and between each of these diseased animals and the WT animal are qualitatively different. For example, $R_{\infty}$ has a sudden increase in the ALS and WT animals, but decreases noticeably in the mdx animals. Moreover, there are substantial differences in the degree that $f_{c}$ changes from baseline in all 3 animal types. In the ALS animals, for example, where there is severe cell atrophy, the elevated $f_{c}$ shows only a relatively small change as compared to that observed in WT animals.

\section{E. Disease states differentiation by the rate of stimulation and animal strength based on the central frequency $f_{c}$ reduction}

Figure 8 (left) shows the relationship between stimulation rate (i.e. strength of induced contraction) and the center frequency $f_{c}$ reduction for all 3 groups of animals. As can be seen WT animals demonstrate a near constant change in center frequency across the stimulation rates, whereas the $\mathrm{mdx}$ and ALS animals both show a much more dramatic relationship, with the reduction in center frequency being far greater with stronger contractions. Such data may reveal important information on the overall ability of a muscle to contract and the neuronal input needed to produce a sufficiently powerful contraction.

Figure 8 (right) shows the relationship between hind limb strength (measured during voluntary contraction) and the change in center frequency with contraction across the entire
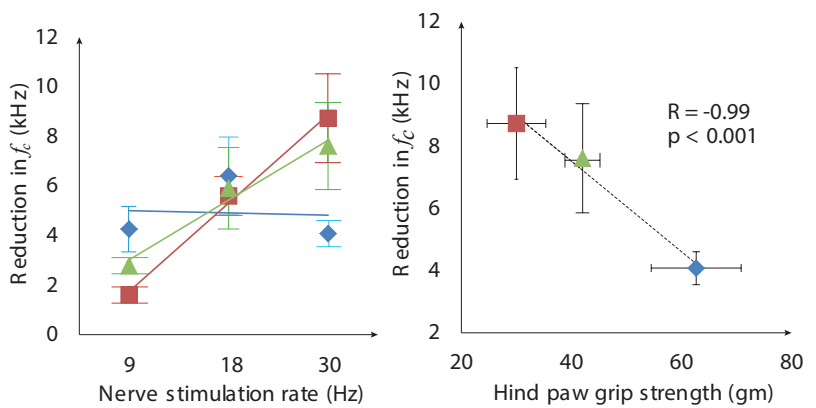

Fig. 8. The reduction in the central frequency $f_{\mathrm{c}}$ depends on the muscle status in WT (blue diamond), ALS (red square) and mdx (green triangle) animals (left). Correlation of the central frequency $f_{\mathrm{c}}$ reduction with animal strength (right). The error bars denote the standard error for all the animals analyzed. The ALS group shows a significantly greater reduction in $f_{\mathrm{c}}$ than wild type animals; No significant difference was found for the mdx animals $(\mathrm{p}=0.04$ and $\mathrm{p}=0.28$, respectively Mann Whitney test, 2 tailed, alpha $=$ $0.05)$

group of animals studied. As can be seen, the weaker animals demonstrate greater changes in center frequency $f_{c}$ than healthier ones, all stimulation parameters being kept the same (30 $\mathrm{Hz}$ stimuli).

\section{DISCUSSION}

Novel multifrequency dynamic-EIM data is reported from a group of healthy and diseased mice during electrically-induced muscle contractions. First, we identified major reductions in 
(A) ALS
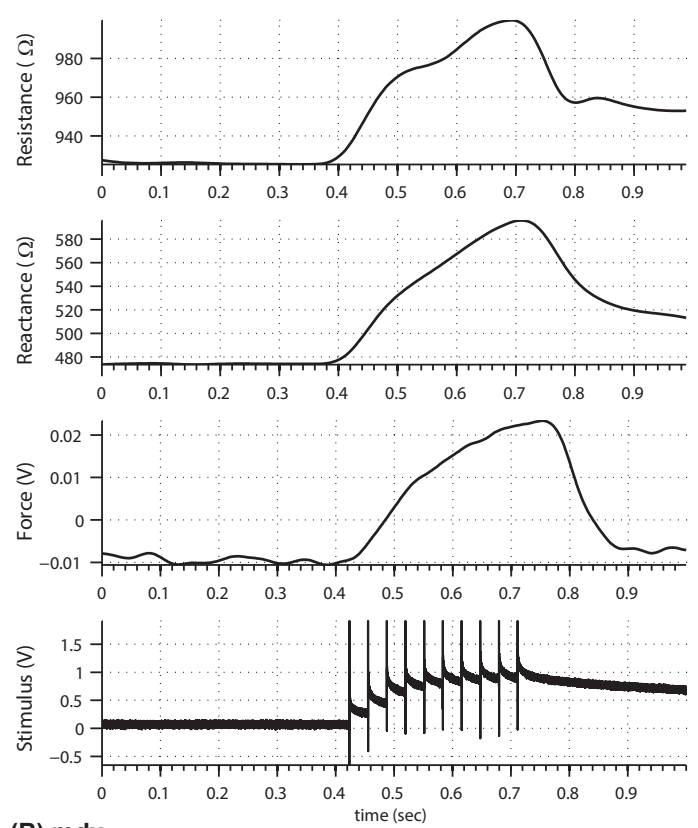

(B) $\mathrm{mdx}$
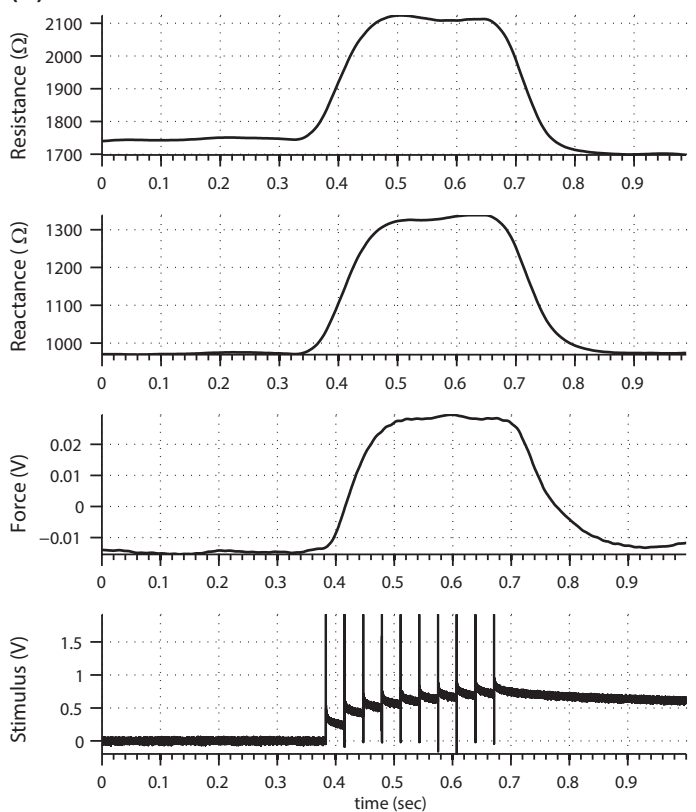
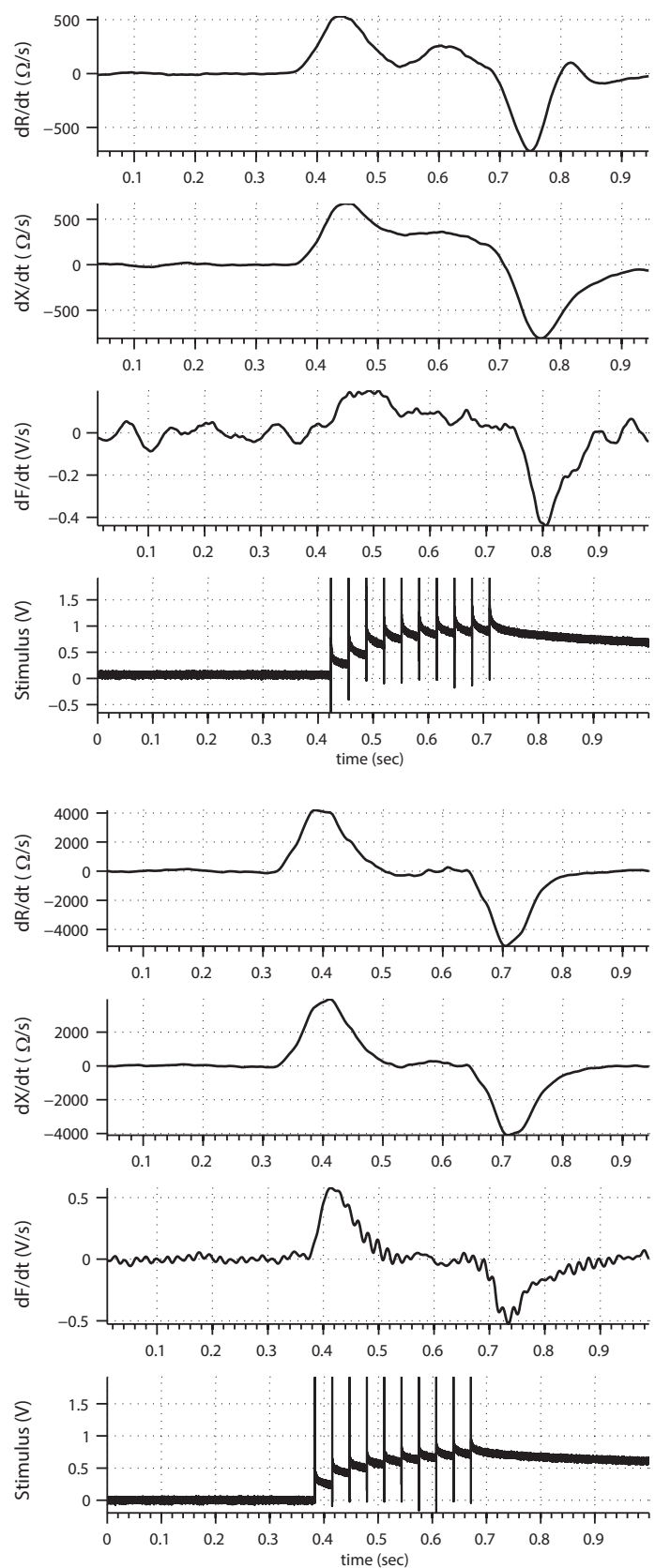

Fig. 6. Time dependences of resistance (R), reactance (X) at $51 \mathrm{kHz}$ and force (F) synchronized with the stimuli for ALS (A) and mdx (B) animal (left) and their corresponding derivatives $\mathrm{dR} / \mathrm{dt}$, $-\mathrm{dX} / \mathrm{dt}$ and $\mathrm{dF} / \mathrm{dt}$ (right). Note differences in scales.

the center frequency $f_{c}$ with contraction in both the WT and diseased animals (Figures 4 and 7), consistent with the concept that during muscle contraction, the muscle fiber diameter size increases slightly. Second, we showed that the Cole parameters do show different temporal behavior in the healthy versus diseased animals (quantified via the derivatives plots shown in Figures 3 and 6). Third, that the diseased animals show a different relationship between force intensity and changes in the Cole parameters (as demonstrated in Figure 8 ) and that muscle strength appears to inversely correlate with the change in $f_{c}$ during contraction, supporting the idea that smaller force changes may be associated with greater impedance changes in diseased states as compared to healthy.

Although the previous single-frequency work done by Shiffman et al in [11] used voluntary isometric contractions in humans and our work utilized involuntary, electrically stimulated isotonic contractions in mice, our findings for the most part are fairly concordant. Namely, we have found (1) that impedance alterations are closely tied to the development of force; (2) that different impedance behaviors are found in diseased as compared to healthy muscle; (3) that there were temporal impedance changes of the tissue post-contraction as compared to pre-contraction, possibly representing some fundamental alteration in the material properties of the muscle 
This article has been accepted for publication in a future issue of this journal, but has not been fully edited. Content may change prior to final publication. Citation information: DOI 10.1109/TBME.2014.2320132, IEEE Transactions on Biomedical Engineering

IEEE TRANSACTIONS ON BIOMEDICAL ENGINEERING

(A) ALS
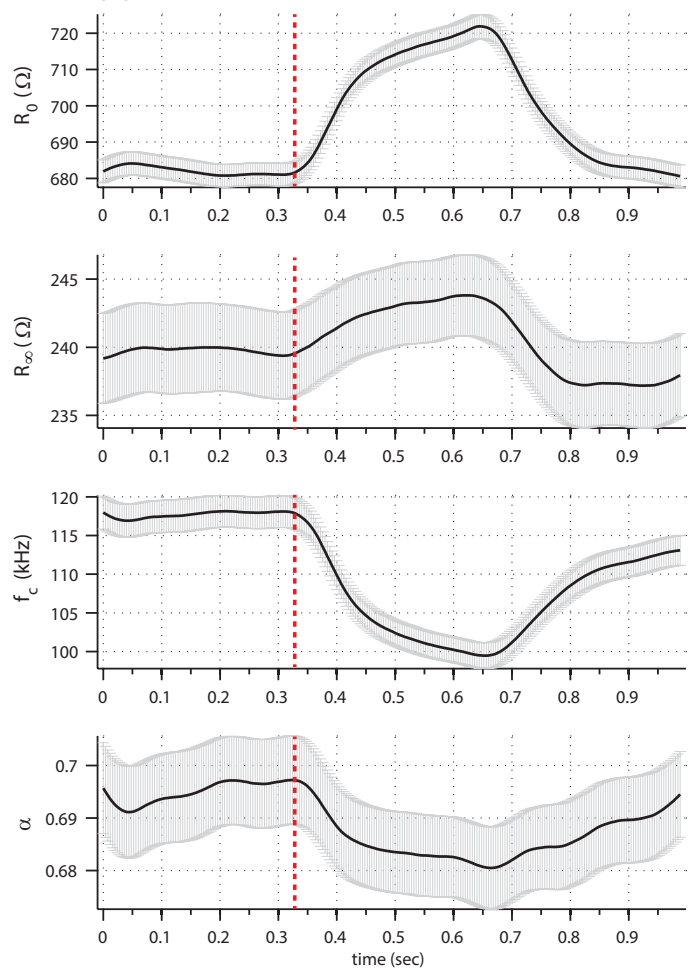

(B) $\mathrm{mdx}$
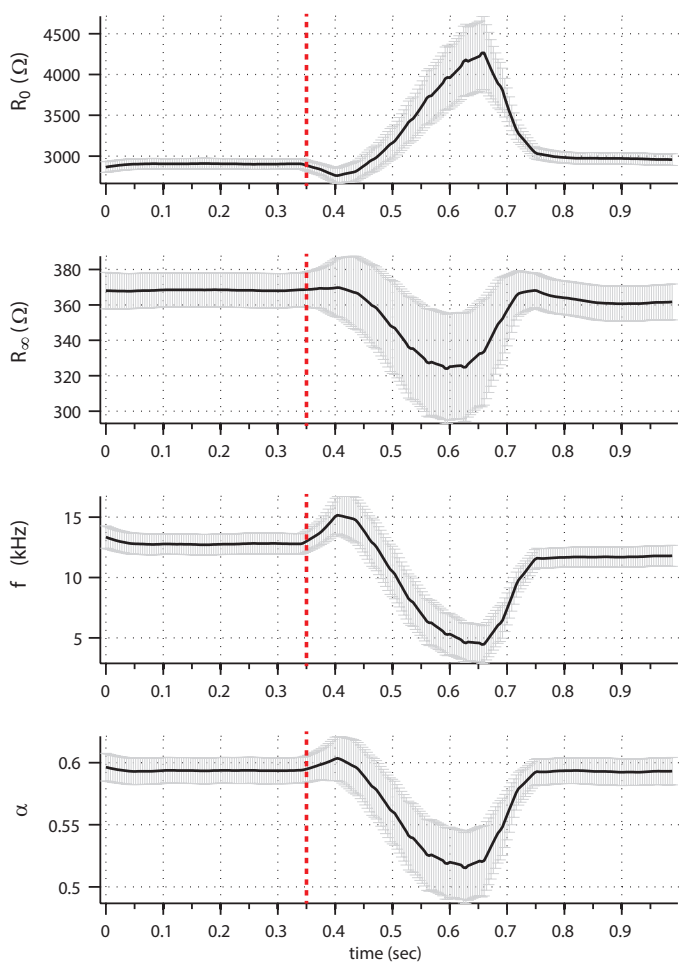

Fig. 7. Evolution of the four estimated Cole impedance model parameters (black) and their standard errors (gray) during contraction-relaxation for ALS (A) and $\mathrm{mdx}(\mathrm{B})$ animal. The red dotted vertical line denote the onset of stimulation.
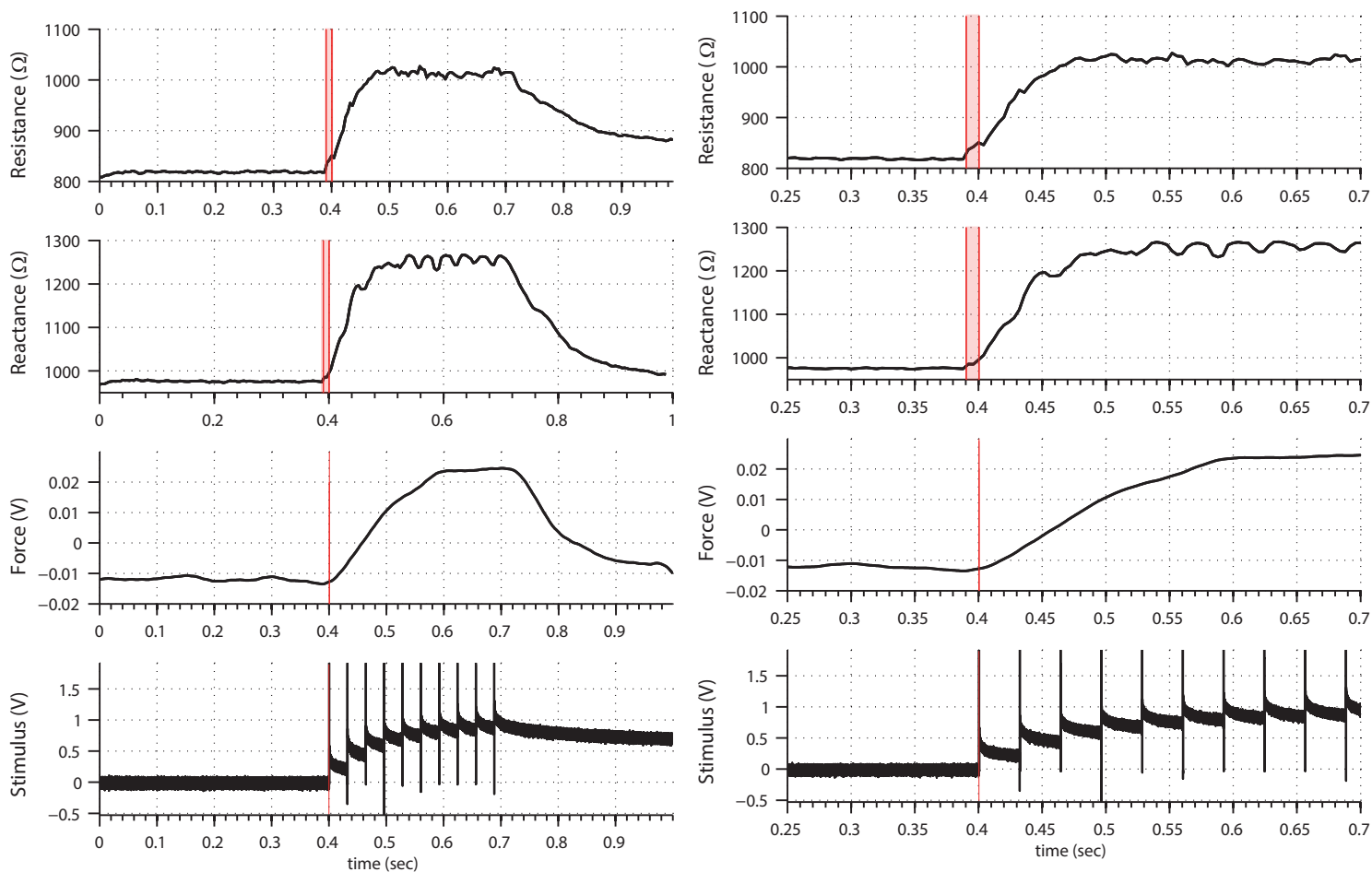

Fig. 9. Temporal dependences of resistance $(\mathrm{R})$, reactance $(\mathrm{X})$ at $51 \mathrm{kHz}$ and force changes (left); High temporal view resolution of the onset changes (right). The red vertical line located at $0.4 \mathrm{sec}$ denotes the beginning of the stimuli and the onset force generation. The red rectangle in $\mathrm{R}$ and $\mathrm{X}$ is the time window $T_{w}=10 \mathrm{~ms}$ duration needed to calculate the impedance spectrum. The reader is referred to Section II for details.

after contraction.

One difference, however, between our results and those earlier ones is that Shiffman et al found that their impedance changes preceded the onset force generation, a complication not easily explained at that time. In fact, we initially also identified a similar finding. However, a close look at our 

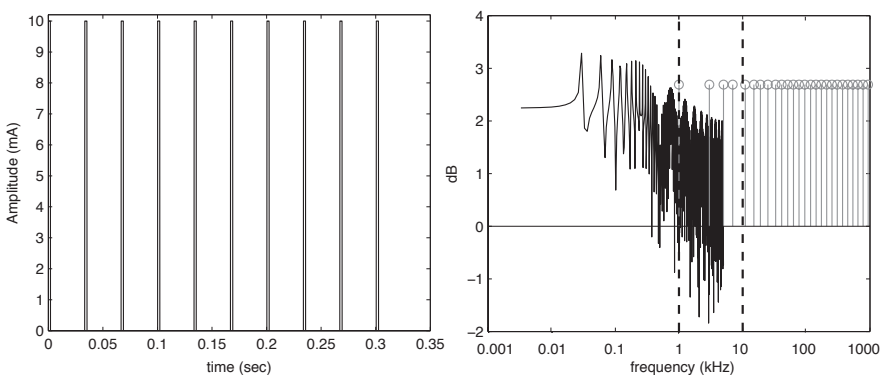

Fig. 10. Time domain simulation of the current stimuli applied to cause strong muscle contraction. Stimuli parameters: number of pulses 10, repetition frequency $30 \mathrm{~Hz}$, pulse duration and amplitude of $0.2 \mathrm{~ms}$ and $10 \mathrm{~mA}$ respectively, sampling frequency 10 ksamples $\mathrm{s}^{-1}$. (left); Magnitude spectra corresponding to the stimuli (black) and multisine (gray) (right). The vertical lines denote the power spectra overlap in the frequency band $1 \mathrm{kHz}-10 \mathrm{kHz}$ between the stimuli and the multisine signal.

data revealed that this perception was due to the temporal window $T_{w}$ needed to discretize data to compute the impedance frequency response using the short time Fourier transform (STFT) [16]. To further prove this, we show Figure 9. Note the red timestamp in the stimuli in which the first stimulus occurred. The reader can observe in the figure that the force exerted by the muscle appears just after the first stimulus, as expected. In terms of impedance, we added as well an additional red time stamp to mark the temporal duration of the STFT window $(10 \mathrm{~ms})$. Thereafter, any onset change in impedance in between these two red stamps, however small and a part from the noise, is because of the overlapping performed when processing data with the STFT (60\%). Thus, contrary to the results reported by the authors in [11], the onset of the impedance change in our data does not precede the onset of the force. We question whether an analogous situation could also have existed in the previous work.

One of the novelties of this research is the combination of synching the complex multifrequency EIM technique and the dynamic changes during muscle contraction. Unlike the current stepped-sine spectroscopy based EIM techniques, the major advantages of multisine technique presented to study dynamic EIM are (i) all EIM spectroscopy data are measured simultaneously and (ii) all EIM spectroscopy data can be gleaned from a single stimulus. The latest is very relevant as repeated stimuli may alter the muscle in complex ways secondary to muscle fatigue.

Nonetheless, there are several technical challenges that deserve further development. One example is coating the electrode surface as well as using flexible electrodes that can deform and follow better the muscle during contraction. Another improvement to pursue would be stimulating the nerve with an arbitrary signal to avoid the actual power spectrum overlap of the stimuli applied with the multisine signal shown in Figure 10. Another avenue we also consider for studying contractile low-frequency impedance data is to induce muscle contraction via chemical means. Additionally, some of the most interesting muscle behavior would be to monitor changes that occur intracelllularly, something that would likely require considerably higher frequencies than those utilized here $(>1 \mathrm{MHz})$. Finally, a detailed evaluation of the effects of anisotropic change in muscle during contraction may also be revealing.

\section{Conclusions}

In this work, we show a complete methodology based on multisine electrical impedance myography (EIM) technique to study the bioimpedance spectroscopic features in healthy and diseased mice during electrical stimulation of the sciatic nerve, with synchronous recording of force and applied stimuli.

The major findings from analyzing the gastrocnemii of 13 mice ( 5 wild type, 4 muscular dystrophy animals, and 4 amyotrophic lateral sclerosis animals) revealed major reductions in the Cole center frequency parameter $f_{c}$ with contraction in both the wild type and diseased animals. Moreover, the Cole impedance model parameters show different temporal behavior in the healthy versus diseased animals. Also of interest, the relationship between force intensity and changes in the Cole impedance model parameters show distinct behavior in the diseased animals.

Spectroscopy dynamic EIM during active muscle contraction have the prospect of revealing new insights into the basic mechanical aspects of muscle behavior during contraction, being an important and useful technique to diagnose neuromuscular diseases where the contractile process is impaired. The major potential advance of this work is that it will allow researchers to measure properties of the muscle most closely aligned to those producing force. To date, no readily quantifiable electrophysiological technique has been available to specifically measure contraction related alterations in the muscle, outside of measuring the force output itself. These impedance-based measures could provide important insights into the mechanism of force reduction that accompanies disorders such as muscular dystrophy and ALS than is possible by simply measuring static data. Such dynamic data may also provide insights into the mechanism of fatigue. Third, and perhaps most importantly, it will allow us to identify and quantify the effect of therapeutic interventions on force production not otherwise captured by standard methodologies. In so doing, it may also provide insights into new potential therapeutic targets.

Future work will focus on further refining the technique, studying a greater number of animals of varying disease severity and type, and correlating alterations in the impedance spectrum to standard functional and histological data.

\section{REFERENCES}

[1] A. Ropper and M. Samuels, Adams and Victor's principles of neurology. New York: McGraw-Hill Professional, 2009.

[2] S. Cirak, L. Feng, K. Anthony, V. Arechavala-Gomeza, S. Torelli, C. Sewry, J. E. Morgan, and F. Muntoni, "Restoration of the dystrophin-associated glycoprotein complex after exon skipping therapy in duchenne muscular dystrophy," Molecular therapy : the journal of the American Society of Gene Therapy, vol. 20, no. 2, pp. 462-7, 2012.

[3] J. Kimura, Electrodiagnosis in diseases of nerve and muscle: principles and practice, 3rd ed. New York: Oxford University Press, 2001.

[4] J. R. Daube and D. I. Rubin, "Needle electromyography," Muscle Nerve, vol. 39, no. 2, pp. 244-70, 2009.

[5] P. W. Hodges, L. H. Pengel, R. D. Herbert, and S. C. Gandevia, "Measurement of muscle contraction with ultrasound imaging," Muscle \& nerve, vol. 27, no. 6, pp. 682-92, 2003. 
[6] S. Rutkove, "Electrical impedance myography as a biomarker for als," Lancet Neurol, vol. 8, no. 3, p. 226; author reply 227, 2009, 1474-4422.

[7] S. B. Rutkove, H. Zhang, D. A. Schoenfeld, E. M. Raynor, J. M. Shefner, M. E. Cudkowicz, A. B. Chin, R. Aaron, and C. A. Shiffman, "Electrical impedance myography to assess outcome in amyotrophic lateral sclerosis clinical trials," Clin Neurophysiol, vol. 118, no. 11, pp. 2413-8, 2007, 1388-2457.

[8] A. W. Tarulli, N. Duggal, G. J. Esper, L. P. Garmirian, P. M. Fogerson, C. H. Lin, and S. B. Rutkove, "Electrical impedance myography in the assessment of disuse atrophy," Arch Phys Med Rehabil, vol. 90, no. 10, pp. 1806-10, 2009.

[9] A. Tarulli, G. Esper, K. Lee, R. Aaron, C. Shiffman, and S. Rutkove, "Electrical impedance myography in the bedside assessment of inflammatory myopathy," Neurology, vol. 65, pp. 451-452, 2005.

[10] S. B. Rutkove, J. B. Caress, M. S. Cartwright, T. M. Burns, J. Warder, W. S. David, N. Goyal, N. J. Maragakis, L. Clawson, M. Benatar, S. Usher, K. R. Sharma, S. Gautam, P. Narayanaswami, E. M. Raynor, M. L. Watson, and J. M. Shefner, "Electrical impedance myography as a biomarker to assess als progression," Amyotrophic Lateral Sclerosis, 2012.

[11] C. A. Shiffman, R. Aaron, and S. B. Rutkove, "Electrical impedance of muscle during isometric contraction," Physiol Meas, vol. 24, no. 1, pp. 213-34, 2003

[12] B. Sanchez, E. Louarroudi, E. Jorge, J. Cinca, R. Bragos, and R. Pintelon, "A new measuring and identification approach for time-varying bioimpedance using multisine electrical impedance spectroscopy." Physiological measurement, vol. 34, no. 3, pp. 339-57, Mar. 2013a.

[13] C. H. Hakim, N. B. Wasala, and D. Duan, "Evaluation of muscle function of the extensor digitorum longus muscle ex vivo and tibialis anterior muscle in situ in mice," Journal of visualized experiments : JoVE, no. 72, 2013.

[14] J. M. Shefner, A. G. Reaume, D. G. Flood, R. W. Scott, N. W. Kowall, R. J. Ferrante, D. F. Siwek, M. Upton-Rice, and J. Brown, R. H., "Mice lacking cytosolic copper/zinc superoxide dismutase display a distinctive motor axonopathy," Neurology, vol. 53, no. 6, pp. 1239-46, 1999.

[15] B. Sanchez, J. Schoukens, R. Bragos, and G. Vandersteen, "Novel Estimation of the Electrical Bioimpedance using the Local Polynomial Method. Application to in-vivo real-time Myocardium Tissue Impedance Characterization during the Cardiac Cycle." IEEE Transactions on Biomedical Engineering, vol. 58, no. 12, pp. 3376 - 3385, 2011.

[16] B. Sanchez, E. Louarroudi, R. Bragos, and R. Pintelon, "Harmonic impedance spectra identification from time-varying bioimpedance: theory and validation." Physiological measurement, vol. 34, no. 10, pp. 12171238, Sep. 2013b.

[17] B. Sanchez, X. Fernandez, S. Reig, and R. Bragos, "A FPGA-based FRA for multisine and stepped sine measurements on stationary and time-varying impedance," Measurement Science and Technology, p. in press, Oct. 2013d.

[18] MATLAB, version 8.0.0.783 (R2012b). Natick, Massachusetts: The MathWorks Inc., 2012.

[19] P. Welch, "The use of fast Fourier transform for the estimation of power spectra: a method based on time averaging over short, modified periodograms," Audio and Electroacoustics, IEEE Transactions on, no. 2, pp. 70-73, 1967.

[20] J. B. Allen and L. R. Rabinier, "Unified approach to short-time fourieranalysis and synthesis," Proceedings of the Ieee, vol. 65, no. 11, pp. $1558-1564,1977$.

[21] R. Pintelon and J. Schoukens, System identification: a frequency domain approach, 2nd ed. IEEE Press, 2012.

[22] K. S. Cole, "Permeability and impermeability of cell membranes for ions," Journal of Chemical Physics, vol. 9, pp. 341-351, 1941.

[23] B. Sanchez, A. S. Bandarenka, G. Vandersteen, J. Schoukens, and R. Bragos, "Novel approach of processing electrical bioimpedance data using differential impedance analysis." Medical engineering \& physics, vol. 35, no. 9, pp. 1349-57, Sep. 2013c.

[24] D. W. Marquardt, "An Algorithm for Least-Squares Estimation of Nonlinear Parameters," Journal of the Society for Industrial and Applied Mathematics, vol. 11, no. 2, pp. 431-441, Jun. 1963.

[25] B. Sanchez, A. Aroul, E. Bartolome, K. Soundarapandian, and R. Bragos, "Propagation of measurement errors through body composition equations for body impedance analysis," IEEE Transactions on Instrumentation and Measurement, vol. PP, no. 99, pp. 1-1, 2013.

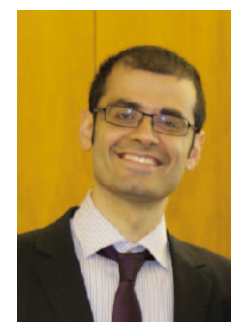

Benjamin Sanchez Terrones was born in Barcelona, Spain, in 1983. He received the M.S. in Telecommunication Eng., the M.S. in Electronic Eng. and the Ph.D. degree in Electronic Eng. from the Universitat Politècnica de Catalunya (UPC), Barcelona, Spain, in 2006, 2007 and 2012 respectively. From 2012 to 2013, he was a Researcher in the Instrumentation and Biomedical Engineering Group from the Electronic Engineering Department at the UPC. Since January 2014, he has been Instructor in Neurology, Harvard Medical School (HMS), Department of Neurology, Beth Israel Deaconess Medical Center (BIDMC). His main interests are the measurement and identification of bioimpedance excited by multisine signals.

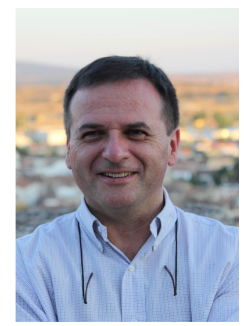

Ramon Bragós Bardia (M'97) was born in Lleida, Spain, in 1962. He received the M.S. Telecommunication Eng. degree in 1991. From 1991 to 1998, he obtained his Ph.D. degree from the Universitat Politècnica de Catalunya (UPC), Barcelona, Spain. Since 1998, he has been associate professor at the Electronics Engineering Department at the UPC. From 2007 to 2009, he was the associate dean of laboratories and, since 2009 , he has been the associate dean of academic innovation at Telecom $\mathrm{BCN}$ school at UPC. His interest is focused on biomedical and biotechnological instrumentation, with emphasis in electrical impedance spectroscopy applications.

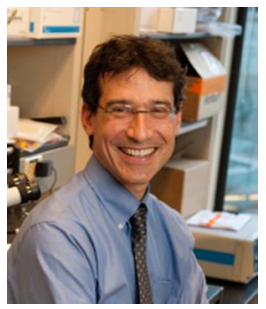

Seward B. Rutkove, MD is Professor of Neurology, Harvard Medical School and Chief, Division of Neuromuscular Disease at Beth Israel Deaconess Medical Center. He has pioneered the development and application of the technique of electrical impedance myography (EIM) for the assessment of neuromuscular disease. In 2011 , he won $\$ 1,000,000$ prize from the non-profit organization Prize4Life, Inc, for the application of EIM technology specifically for the assessment of drug therapies in amyotrophic lateral sclerosis. 